Revue

d'ethnoécologie
Revue d'ethnoécologie

$20 \mid 2021$

Un siècle d'ethno-machins : le centenaire de la Revue de Botanique Appliquée

\title{
Faire face à la crise écologique : l'ethnoécologie comme pratique de connaissance engagée
}

Addressing the environmental crisis: ethnoecology as a committed knowledge practice

Julien Blanc

\section{(2) OpenEdition}

\section{Journals}

Édition électronique

URL : https://journals.openedition.org/ethnoecologie/8741

DOI : 10.4000/ethnoecologie.8741

ISSN : 2267-2419

\section{Éditeur}

Laboratoire Éco-anthropologie

\section{Référence électronique}

Julien Blanc, «Faire face à la crise écologique : l'ethnoécologie comme pratique de connaissance engagée », Revue d'ethnoécologie [En ligne], 20 | 2021, mis en ligne le 31 décembre 2021, consulté le 06 mars 2022. URL : http://journals.openedition.org/ethnoecologie/8741 ; DOI : https://doi.org/10.4000/ ethnoecologie.8741

Ce document a été généré automatiquement le 6 mars 2022.

\section{cc) (†)}

Revue d'ethnoécologie est mis à disposition selon les termes de la licence Creative Commons Attribution - Pas d'Utilisation Commerciale - Pas de Modification 4.0 International. 


\title{
Faire face à la crise écologique : l'ethnoécologie comme pratique de connaissance engagée
}

\author{
Addressing the environmental crisis: ethnoecology as a committed knowledge \\ practice
}

Julien Blanc

\section{Introduction}

1 Il est aujourd'hui commun d'affirmer que la crise écologique que nous vivons a partie liée avec ce que l'on dénomme la modernité. J'en suis moi-même convaincu, comme je suis convaincu qu'en tant que chercheur, universitaire et anthropologue, je me dois de contribuer à y répondre. Les étudiants que je côtoie dans la formation de master à laquelle je prends part me le rappellent d'ailleurs chaque année un peu plus. Travaillé(e)s par ce même sentiment d'urgence qui habite une part croissante des jeunes adultes dans le monde ${ }^{1}$, ils viennent en effet vers notre formation en espérant trouver des réponses à une question qui les taraude, parfois jusqu'à la paralysie: comment faire? Comment à titre individuel, peuvent-ils contribuer à faire face à la crise écologique?

2 Ce sentiment d'urgence, cet appel à agir ici et maintenant, alimente une dynamique dans laquelle je me suis engagé il y quelque temps déjà (2018/2019), poussé par d'autres forces relativement similaires. Celles-là viennent des gens avec lesquels je travaille sur le terrain, agriculteurs ou paysans selon qu'ils se dénomment, car eux aussi m'interpellent sur la manière dont je pourrais, en tant que chercheur, contribuer plus activement à faire face à ce défi et, avec eux, à tenter d'y répondre.

3 Mais comment puis-je contribuer à outiller un peu plus mes étudiants pour à la fois qu'ils se sentent et qu'ils deviennent objectivement plus capables face à la crise ? Et que puis-je faire pour consolider l'entreprise dans laquelle se sont lancés les gens avec 
lesquels je travaille sur le terrain? En d'autres termes, que peut faire un anthropologue de l'environnement et ethnoécologue comme moi pour contribuer à faire face à la crise, une fois l'urgence admise? Que puis-je faire de plus, devrais-je dire, à considérer qu'en faisant mon métier tel que les Institutions m'y incitent, je contribue déjà à apporter des réponses au problème?

Ce texte témoigne du virage que j'ai récemment fait prendre à mes pratiques de recherche dans le but de trouver des réponses, à titre individuel, aux différentes questions posées ci-dessus. Il témoigne des pistes qu'elles me font explorer, sans qu'aucune des réponses apportées jusqu'ici ne soit cependant définitive ou univoque, loin s'en faut. Ce texte n'entend donc pas exprimer ce que devrait être l'ethnoécologie aujourd'hui ou plutôt comment elle devrait se faire. Pour autant, son objectif reste tout de même bien d'en interpeller les acteurs, en particulier face à l'évidence de sa perte de vitesse en France depuis deux bonnes décennies maintenant. Il me semble en effet que notre époque impose à l'ethnoécologie française de s'interroger sur elle-même ; tant sur ses manières de faire science (ses concepts, méthodes et paradigmes) que sur ses manières d'être en société; tant sur ce qu'elle maintient de ses héritages que sur la position qu'elle pourrait prendre aujourd'hui au sein des humanités environnementales.

Comme je vais le préciser dans ce texte, je m'attelle dorénavant à mener une recherche impliquée, proche des principes de la recherche-expérimentation telle que la définit $P$. Nicolas-Le Stratt (2018). Cette manière de faire me conduit à travailler avec les gens qui font mon terrain et non sur eux et d'ainsi participer à la constitution des situations et de l'action sur ces terrains, en l'assumant. Cette manière de faire restitue pleinement aux paysans avec lesquels je travaille leurs qualités de chercheurs. Elle affirme la complémentarité de nos manières d'accéder au monde, de connaître le monde, et cherche à les mettre au travail, ensemble. Mais comme j'en témoignerai ici, adopter cette posture m'a obligé à revoir mes catégories d'analyse, à me défaire de beaucoup des notions sur lesquelles je m'appuyais jusqu'ici. Je traiterai ici en particulier de la manière dont je me suis défait d'une notion pourtant centrale en ethnoécologie, celle de savoirs environnementaux, pour en revenir plus fondamentalement à une interrogation sur ce à quoi renvoie l'acte de connaître.

Le texte qui suit commence par un détour en forme d'hommage à Darrell Posey, personnage que j'ai découvert à travers mes enseignements, qui m'a beaucoup marqué, et a probablement participé à mes changements d'orientation. J'y montre en particulier l'étroite association entre pratiques d'engagement (des chercheurs) et pratique de l'ethnoécologie tout en introduisant des questions épistémologiques qui font le lien avec la partie suivante où j'expose la manière dont je fais aujourd'hui recherche avec des paysans du centre-sud de la France. Le texte en vient alors à expliciter comment, pour mettre en dialogue mes manières de connaître le monde et celles des paysans avec lesquels je travaille, il m'a fallu ouvrir la boîte noire des savoirs environnementaux. Enfin dans la dernière partie, je reviens plus précisément sur les axes de recherche que je développe avec ces mêmes paysans, en les resituant dans les enjeux de connaissance qui traversent aujourd'hui le champ de l'agroécologie et en concluant sur ce que cela implique, à mon sens pour l'ethnoécologie. 


\section{Un petit détour par « l'engagement » comme constante du champ de l'ethnoécologie}

7 À mon arrivée comme enseignant chercheur au Muséum j'ai hérité de l'enseignement d'ethnobotanique dispensé dans le cadre d'un Master alors piloté par plusieurs des membres du laboratoire que j'intégrais ${ }^{2}$. J'ai organisé cet enseignement en grande partie autour de l'intervention de chercheurs et chercheuses installées dans les murs même du Muséum. Elles et ils constituaient en effet un très bon moyen de donner chair au portrait que je tentais de dresser aux étudiants de ce champ de recherche. Mises bout à bout, leurs interventions donnaient à voir les périmètres de l'ethnobotanique et de l'ethnoécologie ${ }^{3}$ et permettaient de discuter ce qui faisait à la fois l'unité et la diversité de ces champs, tant sur le plan des concepts et des méthodes qu'en regard des thèmes traitées (santé et médical, alimentation, agrobiodiversité, etc.). Mais ces interventions constituaient également l'occasion de montrer aux étudiants que beaucoup de ces chercheur(e)s, en même temps qu'ils s'attachaient à décrypter la mécanique des relations entre sociétés et natures, déployaient beaucoup d'énergie à défendre les groupes humains auprès desquels ils travaillaient. Cet engagement envers des collectifs souvent pris dans le jeu de rapports de force écrasant n'était certes pas toujours explicite. Il illustrait cependant une (relative) constante de l'histoire de ce champ, où chercheurs et chercheuses se sont de tous temps attaché(e)s à mettre en lumière l'ingéniosité des gens avec lesquels ils travaillent, la richesse de leurs savoirs environnementaux ou encore leur responsabilité vis-à-vis des êtres et milieux avec lesquels ils composent au quotidien.

8 Je dépeignais ainsi aux étudiants un monde de l'ethnoécologie empreint de figures militantes; des femmes et des hommes qui, soit jouaient de leur appartenance au monde académique pour faire avancer les intérêts de groupes souvent qualifiés de "marginalisés ", soit contribuaient à fomenter des dynamiques d'éducation populaire dans des contextes sociaux dégradés en s'appuyant sur leurs compétences dans ce champ. Parmi tous les exemples que j'évoquais, mon préféré était celui de Darrell Posey. Formé tout à la fois à l'entomologie, l'agronomie, l'anthropologie et la géographie, et pratiquant une ethnobiologie très large (bien que particulièrement compétent en ethnoentomologie), D. Posey travailla au Brésil - avec les indiens Kayapo en particulier - durant la plus grande partie de sa carrière ${ }^{4}$. Il se fit notamment connaitre pour sa contribution à l'établissement de ce qui est considéré comme le premier document international sur les droits des populations autochtones, nommément, la Déclaration de Belem $(1998)^{5}$ aussi connue comme le « code éthique des ethnobiologistes ». Il fut également l'un des personnages clés de la construction de la Société Internationale d'Ethnobiologie (ISE) et l'organisateur d'une exposition qui fit grand bruit lors du sommet de Rio, «La science Kayapo : alternative contre la destruction ». L'histoire raconte ainsi que ses agissements furent décisifs pour l'inclusion des fameux articles $8 \mathrm{j}$ et $8 \mathrm{~d}$ dans la convention sur la diversité biologique ${ }^{6}$. À la fois chercheur et militant, D. Posey produisait du savoir, publiait des articles, coordonnait des ouvrages et défendait les Kayapo; au point d'ailleurs de s'être fait bannir du territoire national brésilien pour avoir réussi à convaincre la Banque Mondiale de se retirer du projet de construction d'un méga-barrage sur le fleuve Xingu (en terre Kayapo).

9 Le personnage de D. Posey m'intéressait également beaucoup pour la définition même qu'il proposait de l'ethnobiologie ou, tout du moins, ce que j'en avais lu et retenu. S'il 
pouvait parfois caractériser l'ethnobiologie comme un champ d'investigation scientifique, D. Posey se référait également parfois à «l'ethnobiologie Kayapo », en tant que système intégré de croyances et de pratiques, en tant que savoir sur les relations entre plantes, animaux et humains. Le groupe d'ethnobiologie qu'il avait constitué au Musée Goldi (à Belem au Brésil) regroupait d'ailleurs un entomologiste, un botaniste, plusieurs ethnologues et géographes, une ethnopharmacologue, mais également des Indiens Kayapo, dotés chacun, comme Posey l'indiquait, de compétences spécifiques et complémentaires. En d'autres termes, D. Posey semblait concevoir l'ethnobiologie avant tout comme une pratique de connaissance - voire de production - du monde (des environnements en particulier) ouverte à toutes modalités et outils d'appréhension, de compréhension, et d'investissement dans ce dernier ; ouverte notamment à des formes d'intelligibilité du monde qui mobilisent à la fois les corps et l'intellect, le sensible, l'émotion et la raison, qui s'appuient sur une expérience tirée à la fois du présent et du passé, qui sont intimement liées aux manières d'habiter et d'investir (en actes et en pensées) ces environnements ; autrement dit à des formes nettement plus épaisses que celles relevant de la pratique scientifique.

En d'autres termes, D. Posey m'intéressait parce que je le concevais comme naviguant entre ce que Bruno Latour (2007) identifie comme le faire science d'un côté, et le faire recherche de l'autre. Il faisait science en traduisant dans l'épistémè scientifique le résultat de ces manières épaisses de connaître (et de pratiquer) le monde, légitimant au passage l'expertise Kayapo sur les milieux et leur gestion durable dans des arènes où seul le savoir scientifique faisait foi. En cela, il répondait aux règles du jeu d'une science en surplomb utilisée (notamment) pour éclairer des modes d'administration par le haut (production de cadres juridiques, construction de politiques publiques, de programmes de conservation, etc.). Il faisait dans le même temps recherche en mettant en dialogue, concrètement, sur le terrain, des manières distinctes de connaître le monde (la science en constituant une parmi les autres) alimentant alors des modes d'administration par le bas (en tant qu'informant des postures radicalement situées et en actes). Je dirais ainsi que Darrell Posey investissait son métier et le monde de différentes manières, à la fois complémentaires et reliées. Cette pluralité de modes d'action lui permettait de circuler dans une grande diversité d'arènes, de faire se multiplier les connexions, de faire se déployer des réseaux d'alliés autour des Indiens Kayapo et de leurs milieux de vie. Elles lui permettaient ainsi d'embrasser les enjeux auxquels étaient confrontés les groupes de Kayapo avec lesquels il travaillait et dont il était profondément solidaire.

\section{Faire recherche ou Faire science ? L'exploration commune pour faire face à la crise}

11 Chercher ensemble: considérer les femmes et les hommes avec lesquels nous travaillons comme des chercheurs à part entière, en tant qu'ils explorent le monde en permanence, qu'ils se mettent et sont mis à l'épreuve dans le cadre de ce processus exploratoire. Chercher ensemble: articuler différentes formes d'intelligibilité du monde pour contribuer à explorer de nouvelles voies; spéculer collectivement et de manière radicalement située sur ce qui est, sur ce qui pourrait être; et faire advenir d'autres possibles, en actes.

Durant de longues années, j'ai travaillé sur la construction d'alternatives agricoles - et alimentaires. Je dénomme ainsi ces dynamiques qui tentent l'écart (parfois petit, 
parfois grand) vis-à-vis de la modernisation agricole, de l'industrialisation des process, de la prise de pouvoir de la rationalité économique sur toute chose. J'ai travaillé sur de telles alternatives en France et au Brésil, à partir desquelles j'ai produit des analyses et des récits destinés pour l'essentiel aux milieux académiques (articles) et à mes financeurs (rapports), qu'ils s'agissent d'agences de recherche, de Ministères, de Régions, ou d'autres représentants des pouvoirs publics. Je tentais dans le même temps de faire en sorte que ces récits puissent faire sens pour les gens dont ils traitaient. Pour les agriculteurs en premier lieu mais aussi pour ces acteurs intermédiaires (comme on les qualifie en sociologie) qui accompagnaient le changement, ou l'incitaient. Dans tous les cas cependant, ce fut de manière secondaire, comme si je leur demandais de lire entre les lignes. Et même si, sur le terrain, j'en venais parfois à sortir de ma réserve, je restais tout ce temps largement pris dans mon éthos scientifique. J'avais appris à faire science en surplomb, à garder mes distances et à ne pas interférer, à révéler les choses pour faire avancer la science et éclairer l'action publique, toutes échelles confondues. J'aspirais en outre à obtenir une place dans l'académie et il me semblait donc qu'il fallait, pour ce faire, embrasser les normes de la profession. En tout cas, telles que je les imaginais. Aujourd'hui je dirais que tout ça manquait quelque peu de "responseabilité ", pour reprendre le terme forgé par D. Haraway (2008), tant vis-à-vis des gens avec lesquels je travaillais que des enjeux plus généraux que nous pose à tous et à toutes la crise écologique : en me maintenant par principe à distance des mondes en devenir au sein desquels j'étais pourtant plongé, je me préservais d'une réflexion sur la manière dont j'aurais pu y prendre part autant que des inconforts inhérents à toute tentative de répondre aux questions qui m'étaient posées; réponses dont on sait qu'elles ne peuvent qu'être partielles, voire parfois même partiales...

13 J'ai depuis décidé de changer mon fusil d'épaule, en prenant l'option de travailler plus franchement avec les gens qui font mes terrains et non plus sur eux. Ou, pour être plus juste, de marier les deux : de travailler sur eux pour travailler aussi, et peut-être même avant tout avec eux, c'est-à-dire en quelque sorte également pour eux. J'ai ainsi tissé une première collaboration, fin 2019, avec un groupe d'agriculteurs français qui se réclament paysans ${ }^{7}$, signifiant par là leur rapport critique au programme de modernisation agricole. Ces femmes et ces hommes maraîchers, arboriculteurs, éleveurs (souvent tout à la fois) qui font groupe par le biais d'une structure associative $\left(\mathrm{SAEL}^{8}\right)$ adossée à un $\mathrm{CIVAM}^{9}$, tentent d'inventer d'autres manières de faire dans leurs champs comme dans les groupes de pairs qu'ils composent ou les relations en partie marchandes qu'ils tissent avec celles et ceux qui consomment leurs aliments. Si la référence à la permaculture opère dans le groupe comme le plus petit dénominateur commun, elle s'inscrit dans un paysage conceptuel beaucoup plus large, où l'on retrouve, sans exclusivité, des références à l'agriculture naturelle (Masanobu Fukuoka ${ }^{10}$ ) ou à la biodynamie (Rudolf Steiner). Plus encore peut-être, c'est le refus des dogmes qui semble caractériser le mieux ces paysans : ils nourrissent la volonté de se réapproprier un pouvoir d'exploration des possibles, un droit à l'expérimentation que l'administration moderne de l'agriculture tend par définition à préempter. Pour eux, l'agriculture est ainsi d'abord une entreprise curieuse et insatisfaite, faite de négociations toujours hésitantes, où se testent les forces et les limites d'un partenariat qui se veut plus « horizontal », avec les autres existants comme avec leurs semblables.

Dans cette aventure collaborative, je m'attache à la fois à faire science et recherche. À partir de l'observation de leurs manières de faire, j'aspire à produire des textes dont la vocation est de circuler dans le monde académique et universitaire et d'y nourrir des 
débats en cours. Mais dans le même temps, je m'attache à produire des formes (orales et écrites principalement) dont ils et elles puissent directement se saisir afin d'avancer dans leurs réflexions et leurs mises en œuvre (j'y reviendrai). Je mobilise également mes réseaux et mes ressources à leur profit, sollicitant d'autres chercheurs, mettant des étudiants au travail. Ensemble, nous œuvrons à donner de la visibilité aux activités du groupe, ma présence contribuant, même modestement, à accroître leur légitimité vis-à-vis des acteurs locaux et régionaux, et à les faire un peu plus prendre au sérieux. L'enjeu est important. Il s'agit de produire un renversement de perspective, de les propulser - eux et leurs pratiques - des marges vers l'avant-garde ; afin de rendre plus envisageable pour certains de s'installer ou de rompre avec l'héritage familial, en trouvant ici un appui technique et humain, un espace d'échange et d'apprentissage ; afin de montrer aux jeunes en formation que d'autres voies sont explorées, et qu'elles peuvent être viables, vivables et de surcroît humainement très riches. Pour ce faire, il faut multiplier les alliances, étoffer et consolider les réseaux, traduire et enrôler, comme l'exprimeraient les sociologues de l'innovation.

Dans l'ensemble, il s'agit donc bien de participer, en l'assumant pleinement, à la constitution des situations et de l'action, à la production des mondes (humains et nonhumains) de ces paysans qui, dès lors que nous travaillons ensemble, deviennent aussi pour partie les miens. Il nous faut dans ce cadre accepter d'intégrer un jeu balbutiant de tâtonnements et d'interpellations croisées, un jeu dans lequel personne (ni moi ni eux) ne souhaite perdre son indépendance. Au contraire même, car c'est dans la mise en tension de ce qui nous distingue, que cette collaboration prend toute sa force. Certes, il faut réussir à accepter l'incertitude de l'intérêt des résultats de la recherche, l'inconfort de la mise à l'épreuve et la possibilité que le contrat qui nous lie se révoque, faute d'intéressement réciproque. Pour autant, dans le cours du processus, la relation se tisse, et avec elle, la confiance, permettant d'accueillir les potentiels ratés et errements de la démarche. Cette posture n'est pas participative, car il ne s'agit pas d'abord de faire science, mais bien de faire recherche. Elle n'est pas non plus uniquement recherche-action, car elle permet dans le même temps d'alimenter des champs de connaissance fondamentaux. Cette posture est simplement celle d'individus qui, chacun, cherchent une place qui fasse sens (une place convenable) relativement à un problème commun, la crise de la modernité, et qui tentent pour eux-mêmes et pour d'autres d'y trouver des réponses (Figure 1). 
Figure 1 : Réunion du groupe SAEL, association de « paysans » installés dans le Limousin

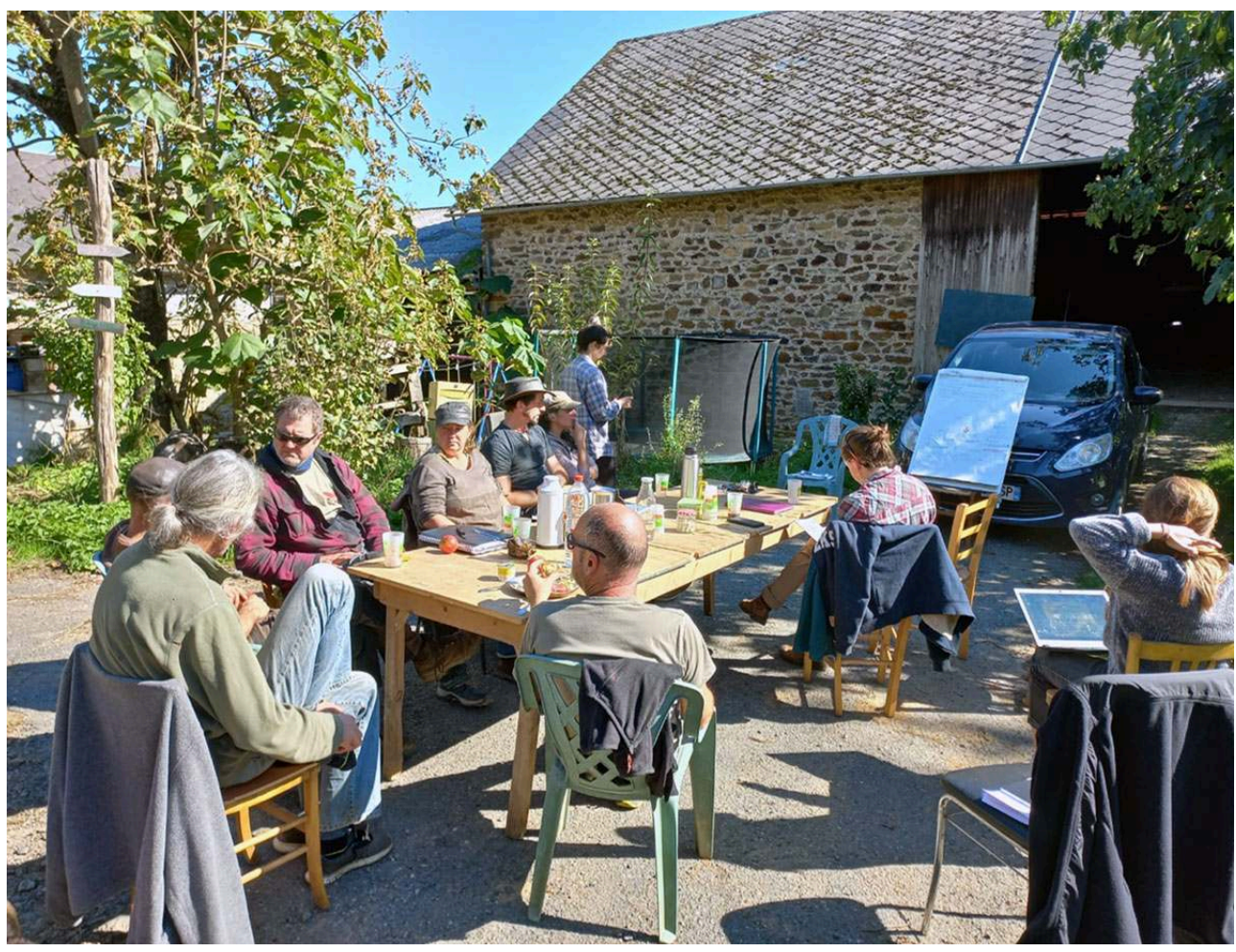

Ici, on parle stratégie de financement du groupe pour les années à venir et priorités en termes d'activités à mener

Photographie J. Blanc

Bien entendu, ce virage a un prix. Celui de faire alliance avec des collectifs choisis parce que leurs orientations et activités font (a priori) largement sens pour moi. Celui de se tourner vers des gens dont je partage, pour partie au moins, les valeurs et l'envie de faire de la micro-politique ; celui de me détourner aussi de la sociologie critique que je pratiquais jusqu'ici ; et, dans mon cas, de me concentrer sur les marges, au détriment du centre, là où se trame pourtant une modernisation écologique qui n'est pas sans poser questions... Mais faire le choix de travailler dans ce cadre constitue pour moi un déterminant important non seulement de la possibilité même de faire recherche mais aussi de réinvestir en son cœur l'ethnoécologie, après un long détour par ses marges.

\section{Rouvrir la boîte noire des « savoirs écologiques / environnementaux »}

17 Je suis aujourd'hui persuadé que pour se donner les moyens d'éclairer des chemins pardelà la modernité, il nous faut non seulement faire une place plus importante aux gens avec lesquels nous travaillons dans le processus de recherche, mais également revoir les termes en lesquels nous avons jusqu'ici posé les problèmes. Dans le cadre de mes travaux sur le déploiement d'alternatives agricoles - et alimentaires, j'ai suivi près de 15 ans durant le fil de la construction et de la circulation des savoirs. À ce titre, je mêlais des entrées classiques en ethnoécologie (intérêts pour les catégorisations, les savoirs environnementaux, etc.) à des cadres d'analyse par ailleurs communément utilisés pour décrire et analyser la production de connaissances en réseau dans le 
domaine agricole ${ }^{11}$. Si je m'intéressais à l'ensemble des acteurs constituant/ construisant ces réseaux et aux interactions qui les structuraient, la symétrie n'a jamais été parfaite et mon attention a toujours été bien plus forte pour les petits agriculteurs avec lesquels je travaillais : pour les transformations de leurs modes de vie, leur façon de travailler et de faire groupe, leurs savoirs et leurs pratiques. Partageant leur quotidien, travaillant dans les champs et les jardins avec eux, c'est toujours d'eux dont j'ai été le plus proche, à eux que je me suis le plus attaché. Pourtant, j'ai toujours eu l'impression de ne pas réussir à témoigner tout à fait fidèlement de ce qu'ils étaient et de ce qu'ils faisaient. Comme si, même à les multiplier, les concepts (ou notions) que je mobilisais, concepts d'interactions, de réciprocité, d'échanges, de domination, d'alliances, de savoirs, de pratiques, de représentations, ne me permettaient ni de saisir (et donc de décrire) pleinement l'épaisseur des relations que les humains avec lesquels je travaillais tissaient entre eux ni avec les non-humains et leurs milieux de vie. Peutêtre était-ce parce que sur les terrains qui étaient les miens, l'individuation des trajectoires et la multiplicité des univers culturels de référence étaient si fortes que ces notions - et les questions qu'elles permettaient de poser n'étaient plus les plus pertinentes ? Ou peut-être est-ce simplement parce que l'expérience que l'on fait des gens est toujours, et de toutes parts, amenée à déborder nos capacités de mise en récit. Dans mon cas, je pense cependant que c'est surtout parce que je suis resté trop pris par l'idée que faire science impliquait de purifier le monde de ses dimensions sensibles et affectives, de traiter de manière objective de la subjectivité humaine.

L'un des développements notionnels propres à l'ethnoécologie fut celui de « Traditional Ecological Knowledge» (TEK) et avec lui ceux de savoirs locaux, autochtones, environnementaux (entre autres). Ces notions ont émergé tardivement dans le champ de l'ethnoécologie/ethnobiologie et celui-ci est loin de s'y laisser résumer. Pourtant, ces notions y ont pris une place centrale dans les années 90, en lien direct avec leur capacité à servir la cause d'une diversité d'acteurs, des groupes autochtones (ou «marginalisés») aux grandes ONGs conservationnistes, et bien d'autres encore, ethnobiologistes inclus ${ }^{12}$. Aujourd'hui, ces notions, et avec elles celles de savoir-faire, de pratiques et de représentations restent, me semble-t-il, assez centrales dans le champ de l'ethnoécologie. Je les ai moi-même utilisées durant de longues années, telles qu'elles m'avaient été léguées et prenant pour acquis leur opérationnalité analytique. Je ne m'étais jamais vraiment interrogé sur ce à quoi elles renvoyaient précisément, ni au rapport qu'elles entretenaient entre elles. Je ne m'étais donc jamais interrogé non plus sur ce que leur usage, tel quel, pouvait oblitérer en retour. Or il me semble que ces notions ne permettent pas de faire recherche tel que je l'ai présenté plus haut et que pour mettre en dialogue, concrètement, différentes manières de connaître le monde, il faut redéplier la notion même de savoirs et, avec elle, celles de pratiques et de représentations. En d'autres termes, il faut s'interroger plus fondamentalement sur ce à quoi connaitre renvoie: dans ses rapports à la pratique (du vivant) comme aux représentations ${ }^{13}$, dans ses dimensions individuelles comme collectives, dans sa part sensible comme intellectuelle. Et cette interrogation doit porter tant sur nos pratiques de connaissance que sur celles des gens avec lesquels nous travaillons.

La notion de savoirs environnementaux a déjà fait l'objet de nombreuses critiques au sein même de la discipline anthropologique. Les premières sont apparues dans le contexte particulier post-Rio 1992, eu égard à leur étroite association aux problématiques de développement et de conservation. La plus célèbre fut sans doute 
celle d'Arun Agrawal (2002) dénonçant la délétère scientisation de ces savoirs : cette opération par laquelle ces derniers se mettent à exister par eux-mêmes, c'est-à-dire en dehors du tissu originel et à chaque fois original de relations dans lesquelles ils sont pris in situ et qui les font concrètement exister ; cette opération qui les discrétise et qui les codifie afin qu'ils puissent répondre aux normes et au fonctionnement de la science (généricité des savoirs, circulation à grande échelle); un processus de pixellisation (Tsing 2012) ou de purification (Latour 1991). De cette critique visant en particulier l'intégration des savoirs autochtones environnementaux dans les grandes bases de données de la Banque Mondiale, émergera le temps d'une interrogation plus large sur ces notions et ce que leur usage oblitère. Maria Carneiro da Cunha ${ }^{14}$ insistera, par exemple, sur le fait que les savoirs environnementaux ne sont que le produit mouvant de "régimes de savoirs $»^{15}$ et que si quelque chose importe c'est de maintenir ces régimes en tant que forces génératives et eu égard à leurs spécificités structurelles. Florence Brunois (2005) proposera, elle aussi, de se détourner des "savoirs locaux" pour s'intéresser aux " modes de connaissances » en eux-mêmes. Là, le regard porte de manière privilégiée sur la façon dont les savoirs sont produits en regard des modes spécifiques d'engagement des humains dans leurs environnements (humains et nonhumains) ; l'enjeu devient donc de réussir à saisir ces modalités d'engagement, à la fois symboliques et pratiques, affectives, sensibles et pragmatiques. En somme, ces chercheurs, comme bien d'autres ${ }^{16}$ avec eux, s'accordent sur l'importance de se détourner d'une conception essentialiste des savoirs et enjoignent à attacher plus d'importance aux processus à l'origine de la connaissance. Les savoirs, nous rappellentils, relèvent d'une pratique qui se déploie dans le cadre de relations, celles que tissent les humains entre eux et avec leurs environnements non-humains (et notamment les êtres qui les composent). S'ils sont parfois stabilisés, s'ils peuvent être codifiés, ils restent fondamentalement mouvants, mis à l'épreuve par un monde lui-même mouvant, inscrits dans des processus génératifs qui les actualisent ou les transforment. Ils relèvent d'une exploration du monde par les corps autant que par l'intellect et qui mobilise le sensible et les affects car ils sont avant tout affaire de relations. Si l'on excepte le contexte du laboratoire, ils se déploient en relation à une « nature » qui n'est pas un substrat mais un partenaire avec qui l'on doit négocier ${ }^{17}$ et se montrer pragmatique. C'est d'ailleurs là l'apport majeur de l'anthropologie, celui de n'avoir de cesse de nous rappeler que nous, modernes, sommes enfermés dans des manières de voir et de faire qui, si elles nous ont indéniablement donné l'avantage sur bien d'autres peuples, nous ont également fermé de nombreuses possibilités de faire monde.

Rouvrir la boîte noire des savoirs, c'est donc comme nous l'écrivions avec Léo Mariani (2022) « réinvestir les domaines que le mouvement de modernisation a forclos, en particulier ces domaines du sensible et de l'expérience que, modernes parmi d'autres, nous avons perdu l'habitude de penser et même de pratiquer » (p. 128). Nous pensons qu'il est important de réapprendre à nous saisir de ces dimensions de la vie dont nous avons probablement minimisé l'enjeu, voire que nous avons contribué à laisser dans l'ombre. Nous en sommes d'autant plus convaincus que l'enjeu est à notre sens à la fois scientifique et politique: tout en ouvrant à d'autres manières de comprendre et de décrire les mondes qui se font, l'opération permet de remettre en question la prévalence du centre sur les périphéries, là où les connaissances dont la science n'arrive pas à se saisir refluent, quand elles n'y sont pas refoulées à dessein. Radicalement situées et fondamentalement relationnelles, celles-là sont inadaptées aux exigences des sciences et de l'ingénierie moderne qui soit ne s'en préoccupe pas, soit de 
façon plus équivoque préfère ne pas les regarder, ne pas voir les questions qu'elles lui posent (Mariani \& Blanc, op. cit.).

\section{Mettre l'ethnoécologie au service de l'agroécologie}

21 L'avènement de l'agroécologie, au début des années 2000, a contribué à faire croître l'intérêt pour les savoirs dit expérientiels, contextualisés ou situés (selon les dénominations) au sein des communautés de chercheurs spécialistes de l'agriculture. En retour, se tisse un programme de recherche ambitieux et exploratoire qui lui aussi cherche à se défaire du tropisme pour la production de savoirs génériques et à réinvestir les dimensions sensibles et relationnelles de la connaissance. Ce programme se nourrit des apports de différents courants scientifiques, dont certains pans des sciences de gestion des connaissances (knowledge management en anglais) qui ont depuis longtemps remis les affects, le sensible et le corps au cœur de leurs travaux (Girard \& Magda 2018, Gherardi 2017). Le lecteur l'aura compris, je suis convaincu que les ethnoécologues disposent également de ressources pertinentes pour nourrir ces perspectives ; moyennant cependant quelques déplacements que j'ai évoqués dans les grandes lignes ci-dessus et que je vais maintenant rapidement détailler, en les ramenant au terrain et en particulier aux travaux que je mène avec le groupe de paysans évoqué plus haut dans ce texte.

La première série de travaux menée avec les paysans du groupe SAEL fut cadrée par une question relativement simple: par quels moyens ces paysans réussissent-ils à travailler selon des formes de contrôle plus souple sur le vivant? Cette question n'est évidemment que simple en apparence, dès lors que la décliner en véritable question de recherche exige de s'intéresser à une multitude d'aspects du métier d'agriculteurs, bien au-delà des frontières des champs ou de la ferme elle-même. Pour commencer, c'est cependant sur les pratiques aux champs que j'ai fait le choix de me concentrer, dans l'idée de tester la manière dont j'étais capable de mettre au travail l'ethnoécologie pour le groupe. Sur le terrain, l'enquête fût prise en charge, sous ma direction, par une étudiante (Djoulia Goueythieu) qui séjourna cinq mois durant chez plusieurs paysans du groupe. D. Goueythieu s'était imprégnée de l'obsession que nous partageons avec Léo Mariani pour la connaissance en pratique et que nous tentons de transmettre aux étudiants dans notre formation ${ }^{18}$. Poussée à faire l'expérience du travail paysan par elle-même durant ces quelques mois, elle vécut et travailla successivement avec quatre membres du groupe, et en tira une ethnographie remarquable.

En matière d'approche, j'étais convaincu qu'il nous fallait commencer par nous défaire des façons les plus communes d'appréhender l'activité agricole, celles qui consistent à se focaliser sur l'action directe volontaire et réfléchie de l'homme sur la matière: agencer des cultures, travailler le sol, semer, tailler, désherber, arroser, etc. ${ }^{19}$. J'encourageais au contraire Djoulia à se focaliser sur de toutes autres pratiques, celles que nous avons fini par regrouper sous le qualificatif de "pratiques attentionnelles ». La littérature générale sur ce thème est abondante et je ne reviendrai pas ici sur la pertinence de son usage. Peut-être est-il simplement nécessaire de souligner que le rôle du corps, que ce soit par les façons de se mouvoir, les gestes techniques ou la mobilisation des systèmes perceptifs, est au cœur de ces pratiques attentionnelles et que les révéler demande des modalités d'enquêtes ad hoc, immersives, participatives et 
appuyées en partie sur l'expérience que le chercheur peut lui-même faire du travail réalisé avec ses enquêtés ${ }^{20}$.

Bien qu'a priori moins visibles que les pratiques d'action directe que j'évoquais plus haut, ces pratiques de nature "attentionnelles » se sont progressivement révélées (dans le cours de l'enquête) comme jouant un rôle fondamental dans le travail réalisé par ces paysans, notamment parce qu'elles déterminaient leur capacité à mettre concrètement en œuvre des formes plus souples de contrôle et de gestion du vivant. En les affûtant, ces pratiques permettent en effet aux agriculteurs de saisir plus finement les manifestations du vivant et d'ajuster toujours mieux les actions qu'ils entreprennent dans leurs champs; d'accroître leur connaissance des causes et des effets en d'autres termes. En effet l'attention est une compétence qui n'est jamais définitivement acquise (contrairement à ce que l'on conçoit comme un savoir), une compétence qui peut toujours s'aiguiser et dont, comme nous le montraient ces paysans, il est possible d'organiser l'amélioration. Ce fut là l'un des principaux fils tirés par Djoulia sur son terrain d'enquête et ce fut à travers lui que nous pûmes dérouler celui du processus de connaissance.

Le résultat de cette exploration fut riche. Il rendit certes compte d'une grande diversité de manières de faire chez ces paysans, mais également de patterns sensiblement identiques chez tous. À ce titre, Djoulia montra en particulier que les agriculteurs produisaient à dessein différents types de dispositifs ${ }^{21}$ visant à produire des situations propices à l'exercice et à l'éducation de l'attention. Certains étaient pensés et mis en œuvre dès la conception du lieu ou du système, d'autres émergeaient au gré de l'expérience, de manière à la fois opportuniste et réflexive. C'est ainsi, par exemple, que certains avaient organisé leurs espaces de travail (répartition des cultures, des zones d'intervention) de telle manière à s'obliger à certains cheminements en particulier : soit pour se contraindre à répéter plusieurs fois les mêmes trajets dans la journée, soit pour s'obliger à emprunter des chemins situés aux marges des espaces les plus travaillés. Si l'on en reste à la question de l'efficacité du travail, le procédé peut paraître étrange car il oblige à des détours. Mais ces détours ont bel et bien une fonction, en offrant à chaque fois l'opportunité d'entendre, de voir et de sentir les manifestations à l'œuvre le long des chemins empruntés. Il invite les paysans à ce que nous avons plus généralement qualifié de détours, gestes - qui s'initient par de nombreux autres biais que celui présenté ici - par lesquels les paysans s'échappent un instant de leur activité du moment (ou de ce qu'ils avaient planifié) pour se rendre attentifs aux manifestations du vivant: on s'arrête ici sur un insecte dont on n'avait jamais repéré la présence aussi tôt (et affirmée) dans l'année, là sur un envahissement herbacé qu'il faudra surveiller de près pour ne pas risquer de se faire dépasser. On développe dans ces instants toute une " éthologie pratique de circonstances " (Mariani \& Blanc à paraître) que seule une attention appuyée aux êtres, aux matières et à leur écologie permet de développer. Il faut prendre son temps, s'obliger à ralentir, contrer les forces qui tendent à accélérer le mouvement, à réduire les enjeux du travail à ceux de productivité, l'enjeu du geste, à celui de son efficacité directe et matérielle. Bien entendu les enjeux de productivité du travail restent fondamentaux, d'autant plus même que ces paysans font le choix de s'outiller de manière très légère, la gestion de la fatigue et de l'usure des corps constituant en retour des problématiques centrales dans la durabilité de ces manières de faire. De sorte que tout l'art de faire de ces paysans semble le mieux se dévoiler dans la manière dont ils cherchent à mettre en balance ces différents enjeux dans les dispositifs techniques comme dans les gestes qu'ils mettent 
en œuvre, dans les choix qu'ils opèrent en matière d'outils comme dans les routines qu'ils choisissent de stabiliser. Précisons que ni cette mise en balance ni cet art de faire ne relèvent de solutions toutes faites, qu'il s'agirait par exemple d'emprunter en des lieux plus éclairés. Rien ici n'est jamais vraiment acquis. Les équilibres comme les manières de faire sont sans cesse mises à l'épreuve. Cet art de faire se cultive dans l'incertitude et relève d'une pratique de connaissance (Figure 2).

Figure 2 : Connaître par le corps

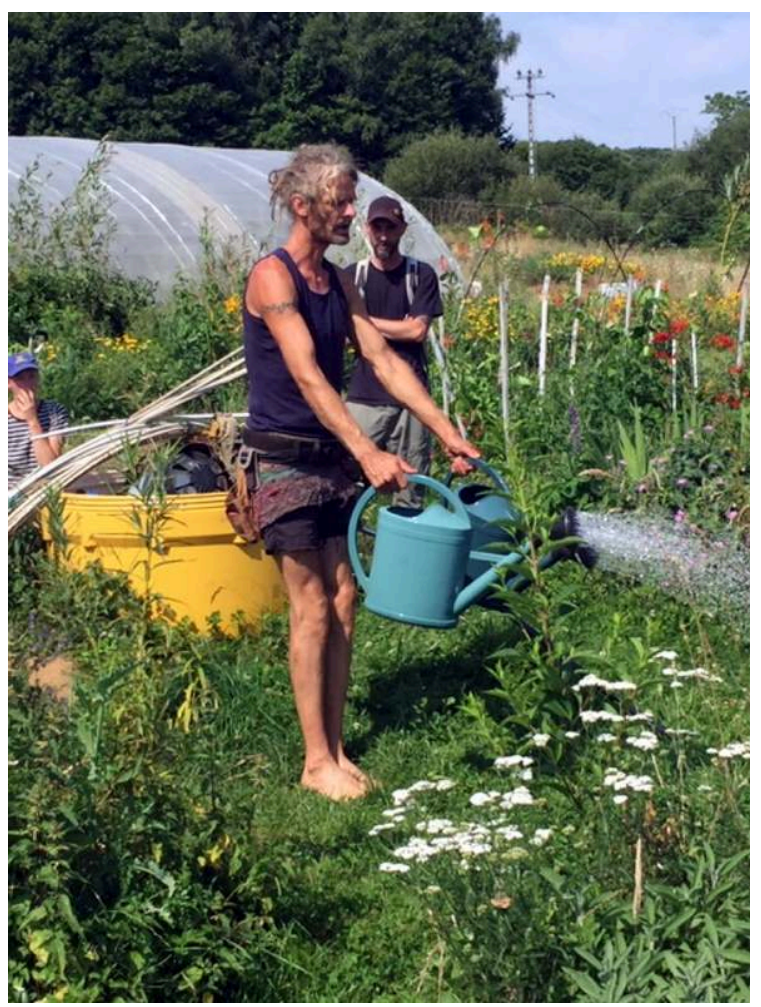

Andy est maraîcher et formateur en permaculture. Lors de son installation il n'a pas investi dans un système d'irrigation, faute de moyens. Cinq ans plus tard, alors qu'il devenait en mesure de le faire, il y a renoncé. Ce renoncement a un prix, celui de passer parfois plus de deux heures par jour à arroser son « jardin ». Pour autant, ce renoncement est aussi un moyen de produire un moment propice à l'observation de ce qui se déroule dans son jardin. Fondamental, dirait-il maintenant pour en assurer un suivi rapproché. Mais l'exercice demande à la fois d'organiser le lieu de manière ad hoc (en distribuant les espaces de stockage de l'eau de manière homogène) et de développer des gestes et des postures adéquates pour ne pas se blesser. C'est ce qu'il est en train de montrer au public en formation sur cette photo.

Déployer une analyse à partir de l'observation de ces "pratiques attentionnelles ", pratiques auxquelles des approches classiques d'agronomie ou de gestion des connaissances resteraient indifférentes, permet ainsi de restituer une tout autre épaisseur au métier d'agriculteur et, par-là, à ce à quoi connaître renvoie dans le cadre de cette activité. Geneviève Delbos l'avait brillamment montré dans son travail sur les paludiers des marais salants de Bretagne-Sud (1983). Comme elle me semble-t-il, on se donne alors les moyens de ne plus considérer l'innovation paysanne comme le résultat mécanique d'une association entre l'expérimentation, les échanges entre pairs et la confrontation aux savoirs livresques (pour simplifier), mais bien de l'interroger comme un processus autrement plus complexe, moins objectivable peut-être, mais plus fondamentalement relationnel aussi. Car l'art de faire que j'évoquais ci-dessus, est bien à la fois, c'est-à-dire dans le même temps, une pratique de connaissance et un art du 
faire relation. Si tel est le cas, c'est qu'il se joue, aussi, et peut-être avant tout dans l'attachement ; aux êtres, aux matières, aux lieux ; qu'il se produit dans l'expérience de l'autre, dans cette curiosité pour l'autre.

Antoine Hennion (2013) insiste sur le fait que l'attention portée aux choses attache. En affirmant cela, il affirme que l'attention constitue également l'un des moteurs du faire relation : elle produit de la relation, crée du lien (Ingold 2000, Despret 2012, Desai \& Smith 2018). Tirer le fil des pratiques attentionnelles tel que nous l'avons fait permet, de fait, de mettre en évidence ce lien quasi mécanique entre ces dernières et la relation que les paysans tissent avec leurs milieux de vie et de travail, ainsi qu'avec certains êtres/matières vivantes dont le statut en vient souvent à se distinguer des autres (certains animaux, les pieds de tomates, certains arbres ou le sol, en particulier). Dans son travail, Djoulia montre ainsi à partir d'exemples précis comment en cultivant la proximité charnelle avec leur environnement, en développant des routines orientées vers le suivi des êtres et des processus vivants, en multipliant les situations dans lesquelles ils doivent faire face aux surgissements impromptus, ces paysans créent les conditions de la rencontre et du dialogue avec les êtres qui peuplent leurs champs et plus largement, qui font leur environnement. Ils créent les conditions d'une capacité à répondre aux besoins de ces êtres, une "response-abilité » comme l'écrit D. Haraway (op. cit.). Non que des savoirs - dans l'absolu - ne puissent être détachés de cette relation, non qu'ils ne puissent servir ailleurs, dans d'autres contextes, mais que parler de savoirs situés, c'est parler dans le même temps d'éthique, une éthique de la responsabilité qui se crée, elle aussi dans la relation et par la relation.

Les résultats de cette recherche ont été mis en discussion avec les membres du groupe à partir de modalités que je ne détaillerai pas ici mais qui furent conçues avec les animateurs du CIVAM et les paysans référents du groupe afin de susciter les échanges et le débat. Il s'agissait d'évaluer ensemble non seulement l'intérêt des résultats mais également la manière dont nous pourrions les mettre au travail, plus avant. Dans l'ensemble, je peux l'affirmer sans ambages, ce que nous avons produit a été très bien reçu par les membres du groupe. Toutes et tous se sont montrés en premier lieu ravis de voir évoquer ainsi leur travail. Nous avions de leur propre avis réussi à en capter des aspects fondamentaux, et pour certains - ceux qui conceptualisaient peut-être moins que d'autres, leurs pratiques - à mettre des mots sur des sentiments, des sensations, des façons de faire qu'eux-mêmes avaient du mal à exprimer. Tous s'accordaient en outre sur la pertinence de donner à lire/voir et à penser autrement leur pratique de ce métier qu'à partir d'entrées techniques ou agronomiques, d'en définir autrement certains des enjeux et des clés de réussite aussi. Je pense que Djoulia a en effet réussi à restituer beaucoup "d'humanité » (pour reprendre les mots de certains paysans) dans le portrait qu'elle a produit de ces paysans et de leur travail; à en "témoigner justement », comme l'exprimerait E. Hache (2011). Si tel est le cas, c'est en partie que nous avons posé d'autres questions à leur métier, utilisé d'autres notions pour l'analyser autant qu'une méthode immersive qui s'est avérée particulièrement féconde. Nous avons par ce biais me semble-t-il réussi à produire les moyens de ne pas séparer $a$ priori les choses: pas d'opposition entre nature et culture, pas de séparation entre savoirs et pratiques, pas de positionnement de l'esprit d'un côté et du corps de l'autre et encore moins de ségrégation des sensibles, de l'affectif et du rationnel : mais une saisie par le mouvement de l'exploration de l'altérité du monde (ici des environnement non-humains) que font ces paysans, de cette pratique de la connaissance, située et 
toujours inachevée, fragile, fractale et résolument pragmatique (elle finit toujours par faire l'épreuve du réel).

En somme, nous avons réussi à les interpeller en leur donnant matière à réfléchir et agir, en pointant vers une nouvelle façon d'identifier ce qui faisait commun dans ce groupe, en ouvrant vers une nouvelle manière de qualifier certains enjeux fondamentaux à la réussite de leur entreprise (telle qu'ils la conçoivent). Partant, plusieurs décisions furent prises, comme celle de construire des journées de formation/ d'échange sur la base de certaines des thématiques creusées, des journées qui seraient ouvertes à un plus large public (de nouveaux installés, d'agriculteurs en réorientation) dans le cadre des activités du réseau CIVAM ; comme celle de produire un document stabilisé à partir de nos apports respectifs sur ces questions, destiné là aussi à circuler dans les milieux professionnels; comme celle enfin, de se lancer dans la construction d'un ouvrage collectif, polyphonique pour être plus exact, constitué de matériaux hétérogènes de sorte que la forme fasse écho au fond: celle d'explorer ensemble, à partir de nos compétences et prises respectives de nouvelles voies; celle donc, comme je l'exprimais en début de texte, de spéculer collectivement et de manière radicalement située sur ce qui est, sur ce qui pourrait être; et faire advenir d'autres possibles, en acte. Voilà où nous en sommes aujourd'hui dans ce faire recherche. Sans compter, il va sans dire, le travail de traduction et mise en visibilité auquel je m'attache à travers la rédaction d'un premier texte destiné au milieu académique ${ }^{22}$ et par le biais de mes enseignements.

\section{Conclusion : Partager des mondes, être en relation}

Le cadre collaboratif que je tisse aujourd'hui avec les paysans du groupe SAEL vise la mise à l'épreuve réciproque de nos savoirs et de nos expériences. Il permet de croiser nos formes respectives d'intelligibilité du monde, de les faire prospérer et de les mettre en dialogue. Il permet de faire avancer mes partenaires dans leurs projets, mais aussi d'affirmer l'intérêt et l'importance de leurs explorations en d'autres arènes autant que de mettre en débat des questions plus générales et fondamentales, qu'elles soient de nature épistémologique, qu'elles interrogent les relations entre sciences et sociétés ou qu'elles portent sur les façons de faire face à la crise. Cette manière de faire à la fois recherche et science oblige à s'inscrire dans les réseaux de relations au sein desquels ces paysans sont eux-mêmes inscrits, à explorer avec eux les relations dans lesquelles ils sont pris. Loin du maintien d'une distance de rigueur prônée parfois en anthropologie, elle nécessite de se donner les moyens d'être au plus proche de leurs pratiques et de leur vécu. Cela implique de partager leur quotidien, de travailler avec eux dans les champs, de les accompagner sur leurs marchés, de participer aux réunions et aux journées de formation, de sorte à éprouver au mieux et avec eux les relations qu'ils tissent avec leurs semblables mais aussi avec les êtres et matières qui peuplent leurs champs. Il faut se laisser prendre par le processus de routinisation, éprouver la fatigue et la lassitude aussi, autant que ces moments d'émerveillement ou de gratification qui émaillent leur quotidien. En partageant cette condition sensible, en se laissant affecter, on tente ainsi de se saisir au mieux de l'épaisseur du vécu, de ce qui meut ces gens, des tensions et des difficultés auxquelles ils font face. En deçà des mots, on cherche à l'éprouver dans nos corps, à se donner les moyens de ne pas se cantonner à un travail sur les représentations, réalisé à partir de l'analyse de discours. Se laisser 
ainsi prendre par son terrain et jouer le jeu des interpellations croisées, c'est considérer que nous, comme eux, sommes conduits à produire un récit situé et partageable (avec eux) sur l'expérience que nous vivons; c'est considérer que nous en venons, nous aussi, à développer des connaissances sur les gens, les situations, les êtres avec lesquels ils et elles travaillent, à partir des prises singulières qui sont les nôtres ; une forme d'expertise que nous allons mettre en commun, avec eux. Mais affirmer cela, n'est-ce pas finalement simplement rappeler, comme tout anthropologue le sait bien, que sa capacité à se laisser prendre (au mieux) dans ces réseaux de relation, à se laisser affecter par son terrain, joue pour beaucoup dans la qualité de son travail ?

Durant de longues années de nombreuses chercheuses et chercheurs ont entretenu l'héritage de l'ethnoécologie au Muséum (Bahuchet \& Lizet 2003, Blanc et al. 2019). Si beaucoup sont aujourd'hui partis ou sur le point de le faire, nous sommes plusieurs chercheurs à toujours y affirmer cette référence ${ }^{23}$. Mais nous sommes peu nombreux et l'héritage se fragilise. De plus en plus, et aussi parce que les étudiants qui suivent nos enseignements le demandent, il me paraît ainsi nécessaire de redéployer cet héritage, de remettre en discussion les catégories, les méthodes et les paradigmes sur lesquels l'ethnoécologie s'est construite. Il est plus que jamais impératif, me semble-t-il, de réussir à démontrer non seulement sa capacité à produire des connaissances mais également à se mettre en société, afin de contribuer à faire face à la crise écologique. J'ai tracé par ce témoignage l'un des chemins qu'il est possible d'emprunter, mais il est loin d'être le seul. Je pense qu'il s'agit avant tout d'une question d'exigence et que chacun est à même, dans cette exigence, d'ouvrir des pistes fécondes. Parfois au risque, certes, d'emprunter des chemins moins reconnus et valorisés par nos institutions. Mais devons-nous nous cantonner à attendre que d'autres que nous prennent ces risques? Ces voies sont par nature risquées et incertaines, mais n'est-ce pas le rôle premier des scientifiques que de s'y engager?

\section{BIBLIOGRAPHIE}

Agrawal A. 2002 - Indigenous knowledge and the politics of classification. International Social Science Journal 54 (173) : 287-297.

Bahuchet S. \& Lizet B. 2003 - L'ethnobotanique au Muséum national d'histoire naturelle. Les hommes, les idées, les structures. In : Plantes, savoirs, symboles. Matériaux pour une ethnobotanique européenne, Actes du séminaire d'ethnobotanique de Salagon. Mane, Musée-conservatoire de Salagon.

Blanc J., Lizet B. \& Juhé-Beaulaton D. 2019 - Un nouvel élan pour l'ethnobotanique au Muséum national d'Histoire naturelle. In : C'est Quoi l'Ethnobotanique? Actes du séminaire d'ethnobotanique de Salagon. Mane, Musée-conservatoire de Salagon.

Brunois F. 2005 - Pour une approche interactive des savoirs locaux : l'ethno-éthologie. Journal de la Société des Océanistes 120-121 : 31-40.

Da Cunha M.C. 2013 - Savoirs autochtones : quelle nature, quels apports? Leçon inaugurale prononcée le jeudi 22 mars 2012. Collège de France. 
Delbos G. 1983 - Savoir du sel, sel du savoir. Terrain 1 : 11-22.

Desai S. \& Smith H. 2018 - Kinship across species: Learning to care for nonhuman others. Feminist Review 118 (1) : 41-60.

Descola P. 2005 - Par-delà nature et culture. Paris, Gallimard, 624 p. (Bibliothèque des sciences humaines).

Despret V. 2012 - Non sheeping sheep. In : Hache (E.) Écologie politique : cosmos, communautés, milieux : 235-271.

Favret-Saada J. 2009 - Désorceler. Paris, Éditions de l'Olivier, 128 p.

Fukuoka M. 2007 - La révolution d'un seul brin de paille. Une introduction à l'agriculture sauvage. Paris, Guy Trédaniel, $202 \mathrm{p}$.

Gély A. 2012 - Darrell Posey : un chercheur engagé. Boletim do Museu Paraense Emílio Goeldi. Ciências Humanas 7 (2) : 581-587.

Gherardi S. 2017 - One Turn... and Now Another One: Do the Turn to Practice and the Turn to Affect Have Something in Common? Management Learning 48 (3) : 345-58. [En ligne] https:// doi.org/10.1177/1350507616688591 Consulté le 10/01/2022.

Gibson J. 1979 - The Ecological Approach to Visual Perception. Boston, Houghton Mifflin.

Girard N. \& Magda D. 2018 - Les jeux entre singularité et généricité des savoirs agro-écologiques dans un réseau d'éleveurs. Revue d'anthropologie des connaissances 12 (2) : 199-228.

Hache E 2011 - Ce à quoi nous tenons : propositions pour une écologie pragmatique. Paris, La Découverte, 247 p. (Les Empêcheurs de penser en rond).

Halloy A. 2006 - Un anthropologue en transe. Du corps comme outil d'investigation ethnographique, In : Noret J. \& Petit P. (Ed.), Corps, performance, religion. Études anthropologiques offertes à Philippe Jespers. Publibook : 87-115.

Haraway D. 2008 - When Species Meet. Minneapolis and London, University of Minnesota Press, $423 \mathrm{p}$.

Hennion A. 2013 - D'une sociologie de la médiation à une pragmatique des attachements. Retours sur un parcours sociologique au sein du CSI. Sociologies. [En ligne] https://doiorg.inee.bib.cnrs.fr/10.4000/sociologies.4353 Consulté le 10/01/2022.

Ingold T. 2000 - The Perception of the Environment. Essays in Livehood, Dwelling and Skill. London \& New York, Routledge, 465 p.

Latour B. 1991 - Nous n'avons jamais été modernes, Essai d'anthropologie symétrique. Paris, La Découverte, $191 \mathrm{p}$.

Latour B. 2007 - L'espoir de Pandore. Pour une version réaliste de l'activité scientifique, Paris, La Découverte, $347 \mathrm{p}$.

López Garcés C.L. \& Robert P. de 2012 - El legado de Darrell Posey: de las investigaciones etnobiológicas entre los Kayapó a la protección de los conocimientos indígenas. Boletim do Museu Paraense Emílio Goeldi. Ciências Humanas 7 : 565-580.

Mariani L. \& Blanc J. 2022 - Conclusion In : Mariani L. (Ed.) Le Goût des possibles. Enquêtes sur les ressorts symboliques d'une crise écologique. Presse Universitaires de Paris Nanterre.

Mariani L. \& Blanc J. à paraître - Dans la texture des relations ; Onto-politiques du vivant dans les nouveaux mondes agricoles, EspaceTemps.net 
Mazabraud B. 2010 - Foucault, le droit et les dispositifs de pouvoir. Cités $42: 127-189$. [En ligne] https://www-cairn-info.inshs.bib.cnrs.fr/revue-cites-2010-2-page-127.htm Consulté le $10 / 01 / 2022$.

Nicolas-Le Strat P. 2018 - Quand la sociologie entre dans l'action. La recherche en situation d'expérimentation sociale, artistique ou politique. Rennes, Éditions du commun, $223 \mathrm{p}$.

Penna-Firme R., de Sá, I.M.M. \& Elisabetsky E.E. 2018 - Remembering Darrell Posey's Contribution to Ethnobiology and Ethnoecology. Ethnoscientia: Revista Brasileira de Etnobiologia e Etnoecologia 3 (2). 10.22276/ethnoscientia.v3i2.138

Posey D.A., Plenderleith K. (Ed.) 2002 - Kayapó Ethnoecology and Culture. NY-London, Routledge, $304 \mathrm{p}$.

Tsing A. Lowenhaup 2012 - On non scalability, Common Knowledge 18 (3) : 505-524. https:// doi.org/10.1215/0961754X-1630424

\section{NOTES}

1. https://www.la-croix.com/Le-sentiment-durgence-climatique-progresse-

monde-2021-01-27-1201137276

2. Richard Dumez, Serge Bahuchet et Marie Roué principalement.

3. J'ai assuré un enseignement d'ethnobotanique quatre années successives avant de le transformer en un enseignement d'ethnoécologie (2018) puis de le requalifier en un enseignement en « anthropologie de l'environnement ».

4. On trouvera un recueil de ses principaux articles réunis par K. Plenderleith dans Posey (2002)

5. Rédigée à la suite du $1^{\mathrm{er}}$ Congrès International d'Ethnobiologie, à Belem en 198, dont il fut l'un des principaux organisateurs.

6. Voir à ce sujet Gély 2012, Lopez Garces \& Robert 2012, Penna-Firme et al. 2018.

7. Je garderai jusqu'au bout du texte la dénomination «paysans" pour qualifier ces femmes et ces hommes qui s'affirment comme tels.

8. Système Agroécologique en Limousin

9. Les Centres d'Initiative et de Valorisation Agricole et du Milieu rural (CIVAM) sont des structures associatives travaillant au développement agricole et rural selon les principes de l'éducation populaire. Il s'agit du CIVAM en Limousin.

10. Sur Fukuoka, voir par exemple Fukuoka 2007.

11. Notamment lié au concept de systèmes de connaissances et d'innovation en agriculture (AKIS en anglais).

12. C'est-à-dire celle de réussir à mettre en commensurabilité des univers de sens et de pratiques distincts, principalement dans le but d'enrôler des partenaires supplémentaires afin d'assurer le déploiement des réseaux et leur stabilisation dans le temps.

13. En tant que systèmes d'interprétation et de modélisation au travers duquel les humains donnent sens aux phénomènes dont ils font l'expérience et qui contribuent à orienter leurs actions.

14. Voir sa leçon introductive donnée au Collège de France en 2012 (Da Cunha 2013).

15. Structurés autour de « schèmes de la pratique » tels que les a définis Philippe Descola (2005)

16. Comme par exemple Tim Ingold, Mark Harris, Carlos Sautchuk, Geneviève Delbos, etc.

17. Florence Brunois, quoi que rapidement, fait référence à ces deux dimensions, celle de la relation négociée et de l'éducation à l'attention (elle cite à ce titre Gibson [1979]). 
18. Intitulée Savoir, pratiquer et éduquer (à) l'Environnement (SPEE), cette unité dispense un enseignement à la croisée de l'épistémologie et de l'anthropologie de l'environnement ; finalité de master 2 Diversité Culturelle, Diversité Biologique (parcours Sociétés et Biodiversité).

19. Ceci pour ce qui concerne les actions dites techniques.

20. Voir Favret-Saada 2009, Halloy 2006, Mariani \& Blanc 2022.

21. Je fais ici référence au dispositif tel que celui défini par Foucault, même si cette référence peut être ramenée à une version «faible ». Pour autant, comme le rappelle Mazabraud (2010), le dispositif foucaldien peut-être défini à partir de 5 principes fondamentaux qui, dans notre cas, semblent opérant : « 1) tout dispositif est une formation historique spécifique ; 2) il met en réseau des éléments hétérogènes ; 3) il a une fonction stratégique dans des relations de pouvoir ; 4) il ouvre corrélativement des champs de savoirs qui codent ou décodent les rapports de pouvoir ; 5) le dispositif est remobilisé pour gérer les effets qu'il a lui-même produit, que ce soient des résistances, des détournements, ou d'autres phénomènes de marges. » (in Mazabraud, op. cit. p. 130)

22. Texte où D. Goueythieu et L. Mariani figurent respectivement en deuxième et troisième auteurs, en regard de leurs contributions respectives et soumis à la revue Agriculture and Human Value en mars 2022.

23. Nous faisons notamment groupe autour de cette bannière, comme en témoigne l'intitulé de l'équipe au sein de laquelle nous sommes rassemblés: "Ethnoécologie: savoirs, pratiques, pouvoirs ", dans l'UMR Eco-anthropologie.

\section{RÉSUMÉS}

Ce texte est le témoignage d'un enseignant-chercheur, ethnoécologue, qui s'interroge sur les implications de la montée en puissance de l'urgence écologique sur ses pratiques de recherche. C'est aussi le témoignage d'une trajectoire personnelle marquée par un appel à faire recherche avec les gens et non plus uniquement sur eux. Ce texte est également le témoignage d'une volonté d'explorer des façons de mettre au travail ensemble différentes manières de connaitre et d'agir le monde dans le but de contribuer à renforcer nos capacités à faire face à la crise écologique. C'est enfin un texte qui témoigne d'une nécessaire rupture épistémologique qui seule permet d'interroger plus fondamentalement les processus de connaissance et rend possible cette perspective collaborative. Les pistes que je dessine ici sont toutes aussi situées et inachevées que celles qu'élaborent mes partenaires de recherche sur le terrain. En cela, elles ne prétendent en rien affirmer ce que l'ethnoécologie devrait être ; seulement illustrer la manière dont je tente aujourd'hui, avec les ressources qui sont les miennes, de répondre à cet appel à la responseabilité en provenance du terrain et des salles de classe où j'exerce.

This text is a testimony; that of a university member, an ethnoecologist, wondering about the implications of the growing ecological emergency on his research practices. It is the account of a personal path marked by a call to do research with people and not only about them. It is also the expression of a will to explore different ways to know and act in the world in view to strengthen our ability to face the ecological crisis. Finally, it is the expression of a need for an epistemological break, which could lead to question knowledge processes and make this collaborative perspective possible.

The answers I provide here are just as situated and unfinished as the ones developed by my 
research partners on the field. In this respect, they do not claim to affirm what ethnoecology should be; they merely illustrate the way in which I am trying today, with the available resources, to respond to this call for response-ability, emerging both from the field and from the classrooms where I teach.

\section{INDEX}

Keywords : environmental anthropology, epistemology, agroecology, environmental knowledge, collaborative research

Mots-clés : anthropologie de l'environnement, épistémologie, agroécologie, savoirs environnementaux, recherche impliquée

\section{AUTEUR}

\section{JULIEN BLANC}

Maître de conférences UMR 7206 Muséum national d'Histoire naturelle 